\title{
Just us boys : diversity in the GNU project as measured by mailing list activity.
}

\author{
Camille Akmut
}

October 4, 2019

\begin{abstract}
Structure and patterns of a male-dominated culture. Statistics measured over a period of one year (leading up to, including September 2019).
\end{abstract}




\section{Introduction : GNU is not...}

In the following we measure diversity in the GNU project by using mailing list activity, as proxy ${ }^{1}$.

Specifically, we chose info-gnu, where announcements relating to the GNU project are posted.

All the major figures (e.g. Richard Stallman, Werner Koch, Alexandre Oliva...) of this free software project - arguably the most important of all - make regular appearances here and release information regarding their respective areas, or software.

These encompass the well-known ones : GNU itself, GPG, Linux-Libre, GCC, GDB, coreutils, etc.

GNU is not for women - such would be the conclusion of anyone going through our statistics.

And, if the mostly passive attitudes adopted so far have led to such results, surely they can no longer be considered enough.

At the turn of the last century, men formed peculiar circles, still, in and around universities.

The more prestigious these places, the longer it took them to admit women inside their walls - and longer still to admit them as equals. ${ }^{2}$

In the novels of Waugh and Forster, where the old, male-exclusive university system still exists, undisturbed by the advances of times, the absence of women goes largely unnoticed : unproblematic.

"This is just how things are", "They've always been this way" - so went their inner lives.

Lise Meitner (who ended up finding a place within academia) or Emmy Noether had no place in these novels, no more than they had in the times they depict...

As to the satisfaction of their pleasures, these gentlemen, knowledgeable as they were about ancient Greek texts, had long found solutions of their own.

To heterosexual casuistics ${ }^{3}$ of old days correspond now intricate rationalizations to make up for the absence of women and minorities of various kinds :

They blame schools, they blame society, even the weather or the stars if they could - so long as not themselves.

And, so, they continue to live peaceful lives, not unlike the eternal Cambridge and Oxford of aforementioned novels... "Just us boys" is their motto - now as it was then.

\footnotetext{
${ }^{1}$ Other proxies (i.e. approximations) can be used.

${ }^{2}$ This is consistent with phenomena in other areas; the greatest inequalities are usually found at the top, never at the bottom : board of directors, executives and CEO's, software engineer positions, etc.. Or project leaders, as in this case.

${ }^{3}$ Casuistics were (largely obscure, if not irrational) conditional statements that formed complex moral and philosophical systems amongst Medieval scholars such as those of Christianity - though, certainly, not only them.
} 


\section{Raw data}

\begin{tabular}{|c|c|c|}
\hline Project & Author & Gender \\
\hline - September 2019 & & \\
\hline $\begin{array}{l}\text { GNU Project } \\
\text { Texinfo } \\
\text { GDB } \\
\text { GNU Datamash } \\
\text { GNU Linux-libre } \\
\text { Bison } \\
\text { GNU Mes } \\
\text { GNU Wget2 } \\
\text { GNU gama }\end{array}$ & $\begin{array}{l}\text { Richard Stallman } \\
\text { Gavin Smith } \\
\text { Joel Brobecker } \\
\text { Assaf Gordon } \\
\text { Alexandre Oliva } \\
\text { Akim Demaille } \\
\text { Jan Nieuwenhuizen } \\
\text { Tim Ruhsen } \\
\text { Ales Cepek }\end{array}$ & $\begin{array}{l}\mathrm{m} \\
\mathrm{m} \\
\mathrm{m} \\
\mathrm{m} \\
\mathrm{m} \\
\mathrm{m} \\
\mathrm{m} \\
\mathrm{m} \\
\mathrm{m}\end{array}$ \\
\hline - August & & \\
\hline $\begin{array}{l}\text { GNU findutils } \\
\text { Libgcrypt } \\
\text { Emacs } \\
\text { nano } \\
\text { FreeIPMI } \\
\text { GNU Scientific Library } \\
\text { GCC } \\
\text { Unifont } \\
\text { Gnuastro } \\
\text { GNU C Library }\end{array}$ & $\begin{array}{l}\text { Bernhard Voelker } \\
\text { Werner Koch } \\
\text { Nicolas Petton } \\
\text { Benno Schulenberg } \\
\text { Albert Chu } \\
\text { Patrick Alken } \\
\text { Jakub Jelinek } \\
\text { Paul Hardy } \\
\text { Mohammad Akhlaghi } \\
\text { Carlos O'Donell }\end{array}$ & $\begin{array}{l}\mathrm{m} \\
\mathrm{m} \\
\mathrm{m} \\
\mathrm{m} \\
\mathrm{m} \\
\mathrm{m} \\
\mathrm{m} \\
\mathrm{m} \\
\mathrm{m} \\
\mathrm{m}\end{array}$ \\
\hline- July & & \\
\hline $\begin{array}{l}\text { GNU Stow } \\
\text { Denemo } \\
\text { GNU direvent } \\
\text { rush } \\
\text { GnuPG } \\
\text { GNU Linux-libre } \\
\text { GNU gama } \\
\text { pies } \\
\text { rush }\end{array}$ & $\begin{array}{l}\text { Adam Spiers } \\
\text { Richard Shann } \\
\text { Sergey Poznyakoff } \\
\text { Sergey Poznyakoff } \\
\text { Werner Koch } \\
\text { Alexandre Oliva } \\
\text { Ales Cepek } \\
\text { Sergey Poznyakoff } \\
\text { Sergey Poznyakoff }\end{array}$ & $\begin{array}{l}\mathrm{m} \\
\mathrm{m} \\
\mathrm{m} \\
\mathrm{m} \\
\mathrm{m} \\
\mathrm{m} \\
\mathrm{m} \\
\mathrm{m} \\
\mathrm{m}\end{array}$ \\
\hline - June & & \\
\hline $\begin{array}{l}\text { GNU Guile } \\
\text { GNU Stow } \\
\text { Nettle } \\
\text { Nettle }\end{array}$ & $\begin{array}{l}\text { Ludovic Courtes } \\
\text { Adam Spiers } \\
\text { Niels Moller } \\
\text { Niels Moller }\end{array}$ & $\begin{array}{l}\mathrm{m} \\
\mathrm{m} \\
\mathrm{m} \\
\mathrm{m}\end{array}$ \\
\hline
\end{tabular}


GNU APL

mailutils

GNU Guile

Gawk

nano

Dr. Geo

GNU Artanis

IceCat

Unifont

- May

GnuPG

GNU OrgaDoc

GNU libidn2

Bison

GNU Guix

Bison

GNU gettext

GNU Mcron

Unifont

GNU Shepherd

GDB

GNU gettext

GNU CSSC

LibreJS

GNU Linux-libre

GCC

GNU Guix

Emms

\section{- April}

dico

nano

rush

GNU Shepherd

Gnuastro

nano

Emacs

Gawk

wget

wget

- March

GnuPG

nano

GnuPG

LibreJS
Dr. Jurgen Sauermann m

Sergey Poznyakoff m

Ludovic Courtes m

Arnold Robbins m

Benno Schulenberg m

Hilaire Fernandes m

Nala Ginrut [Lei Mu] (?)

Ruben Rodriguez m

Paul Hardy m

Werner Koch m

Adam Bilbrough m

Tim Ruhsen m

Akim Demaille m

Ludovic Courtes m

Akim Demaille m

Bruno Haible m

Adam Bilbrough m

Paul Hardy m

Ludovic Courtes m

Joel Brobecker m

Bruno Haible m

James Youngman m

Ruben Rodriguez m

Alexandre Oliva m

Jakub Jelinek m

Ludovic Courtes m

Yoni Rabkin m

Sergey Poznyakoff m

Benno Schulenberg m

Sergey Poznyakoff m

Ludovic Courtes m

Mohammad Akhlaghi m

Benno Schulenberg m

Nicolas Petton m

Arnold Robbins m

Darshit Shah m

Darshit Shah m

Werner Koch m

Benno Schulenberg m

Werner Koch m

Ruben Rodriguez m 


coreutils
Unifont
GNU Linux-libre
GNU Octave
- February
GNU Dr. Geo
GNU ddrescue
dico
tar
mailutils
cflow
GCC
GNU MCSim
FreeDink
Texinfo
GnuPG
GNU libidn 2
GNU gama
GNU Binutils
- January

Padraig Brady m

Paul Hardy m

Alexandre Oliva m

John W. Eaton m

Hilaire Fernandes m

Antonio Diaz Diaz m

Sergey Poznyakoff m

Sergey Poznyakoff m

Sergey Poznyakoff m

Sergey Poznyakoff m

Jakub Jelinek m

fredomatic [Frederic Bois?] m

Sylvain Beucler m

Gavin Smith m

Werner Koch m

Tim Ruhsen m

Ales Cepek m

Nick Clifton m

$\begin{array}{lll}\text { GNU MPFR } & \text { Vincent Lefevre } & \mathrm{m} \\ \text { GNU C Library } & \text { Siddhesh Poyarekar } & \mathrm{m} \\ \text { Bison } & \text { Akim Demaille } & \mathrm{m} \\ \text { Bison } & \text { Akim Demaille } & \mathrm{m} \\ \text { FreeIPMI } & \text { Albert Chu } & \mathrm{m} \\ \text { FreeDink } & \text { beuc [Sylvain Beucler ] } & \mathrm{m} \\ \text { GNU Ocrad } & \text { Antonio Diaz Diaz } & \mathrm{m} \\ \text { GNU MDK } & \text { Jose A. Ortega Ruiz } & \mathrm{m} \\ \text { Readline } & \text { Chet Ramey } & \mathrm{m} \\ \text { Bash } & \text { Chet Ramey } & \mathrm{m} \\ \text { GNUstep } & \text { Ivan Vucica } & \mathrm{m} \\ \text { GNUstep } & \text { Ivan Vucica } & \mathrm{m} \\ \text { GNUstep } & \text { Ivan Vucica } & \mathrm{m} \\ \text { autoconf } & \text { Peter Simons } & \mathrm{m} \\ \text { GNU libidn } & \text { Tim Ruhsen } & \mathrm{m} \\ \text { GNU moe } & \text { Antonio Diaz Diaz } & \mathrm{m} \\ \text { GNU ed } & \text { Antonio Diaz Diaz } & \mathrm{m} \\ \text { recutils } & \text { Jose E. Marchesi } & \mathrm{m} \\ \text { tar } & \text { Sergey Poznyakoff } & \mathrm{m} \\ \text { diffutils } & \text { Jim Meyering } & \mathrm{m}\end{array}$

[Note : Lei is given to both men and women] 


\section{Statistics}

\begin{tabular}{|c|c|c|}
\hline Month (2019) & Male participants & Female participants \\
Sep. & $100 \%$ & $0 \%$ \\
Aug. & $100 \%$ & $0 \%$ \\
July & $100 \%$ & $0 \%$ \\
June & $100 \%(?)$ & $0 \%(?)$ \\
May & $100 \%$ & $0 \%$ \\
Apr. & $100 \%$ & $0 \%$ \\
Mar. & $100 \%$ & $0 \%$ \\
Feb. & $100 \%$ & $0 \%$ \\
Jan. & $100 \%$ & $0 \%$ \\
\hline
\end{tabular}

Table 1: Diversity in the GNU project and gnu-info mailing list participation.

\section{Bibliography}

info-gnu. https://lists.gnu.org/mailman/listinfo

\section{Announcing future research}

-. (upcoming). "Yann Le Cun : portrait of a small-time engineer."

Note : from the very bottom of French engineer schools crochetted his way to the top of international scientific society!

-. (upcoming). "Danah Boyd never bothered anyone. Science in the interest of the rich and mighty."

Note : celebrated by the EFF and employed by Microsoft, but "famous artists are quickly forgotten" according to Peter Doig.

-. (upcoming). "Saint Sheryl."

Note : on Sheryl Sandberg, and more on corporate diversity. Dumb like her barbies - said someone I know... 